Article

\title{
Prevalence, Population Diversity and Antimicrobial Resistance of Campylobacter coli Isolated in Italian Swine at Slaughterhouse
}

\author{
Guido Di Donato ${ }^{1}$, Francesca Marotta ${ }^{2, *}$, Roberta Nuvoloni ${ }^{3}$, Katiuscia Zilli ${ }^{2}$, Diana Neri ${ }^{2}$, \\ Daria Di Sabatino ${ }^{1}$, Paolo Calistri ${ }^{1}$ and Elisabetta Di Giannatale ${ }^{2}$ \\ 1 Istituto Zooprofilattico Sperimentale dell'Abruzzo e del Molise "G. Caporale", National Reference Centre for \\ Veterinary Epidemiology, Programming, Information and Risk Analysis, 64100 Teramo, Italy; \\ gu.didonato@izs.it (G.D.D); d.disabatino@izs.it (D.D.S); p.calistri@izs.it (P.C.). \\ 2 Istituto Zooprofilattico Sperimentale dell'Abruzzo e del Molise "G. Caporale”, National Reference \\ Laboratory for Campylobacter, 64100 Teramo Italy; k.zilli@izs.it (K.Z.); d.neri@izs.it (D.N.); \\ e.digiannatale@izs.it (E.D.G.) \\ 3 Department of Veterinary Sciences, University of Pisa, Pisa, Italy; roberta.nuvoloni@unipi.it \\ * Correspondence: f.marotta@izs.it
}

Received: 3 January 2020; Accepted: 5 February 2020; Published: 7 February 2020

\begin{abstract}
Campylobacter spp. are among the microorganisms most commonly associated with foodborne disease. Swine are known to be the main reservoir of Campylobacter coli and a possible source infection of humans as a result of carcass contamination at slaughter. The aim of this study was to evaluate the prevalence of $C$. coli contamination in swine carcasses, the antimicrobial resistance (AMR) patterns of isolates and the genetic diversity between strains obtained from swine and those isolated from humans. The prevalence of contamination was higher on carcasses $(50.4 \%)$ than in faeces (32.9\%). The 162 C. coli isolated from swine were examined by pulsed-field gel electrophoresis (PFGE) and multi-locus sequence typing (MLST). The results of PFGE indicated a high genetic diversity among the isolates, with 25 different PFGE types. MLST assigned 51 sequence types (STs) to isolates. The most common genotype was ST-854 (16.04\%), ST-9264 (10.49\%) and ST-1016 (6.08\%). Results of AMR showed a high resistance to quinolones and fluoroquinolones together with aminoglycosides and tetracycline. Many strains were multi-resistant with predominant R-type TeSCipNa (57\%). Five resistance genes were detected along with mutation in the gyrA gene. A strong correlation between phenotypic and genotypic resistance was found for fluoroquinolone and tetracycline. Genetic profiles obtained in swine isolates were compared to those of 11 human strains. All human strains and $64.19 \%$ of animal strains (104/162) were assigned to the ST-828 clonal complex.
\end{abstract}

Keywords: Campylobacter coli; molecular analysis; antimicrobial resistance

\section{Introduction}

Campylobacter spp. are among the most common causes of bacterial diarrhoea worldwide and are estimated to cause approximately 246,000 illnesses annually in the European Union (EU), mostly due to consumption of contaminated food [1]. Campylobacter may be transferred to humans indirectly through the ingestion of contaminated water or food [2] and less frequently by direct contact with contaminated animals or animal carcasses. The species most commonly associated with human infection are Campylobacter jejuni followed by Campylobacter coli and Campylobacter lari, although other Campylobacter species, including the non-thermophilic Campylobacter fetus, are known to occasionally cause human infection [1]. 
C. jejuni is considered the most frequent Campylobacter species associated with disease in humans, and are responsible for about $80 \%-90 \%$ of the total number of human cases of campylobacteriosis in the EU [1]. However, different studies have highlighted the importance of C. coli as an emergent problem in public health due to its greater resistance to antibiotics [3,4]. In the EU, C. coli has been found to be responsible for about $9 \%$ of human campylobacteriosis in the EU. Food producing animals like poultry, cattle and swine are common hosts and important reservoirs of Campylobacter species. C. jejuni is considered prevalent in poultry [5] and cattle [6], while pigs are mostly implicated as reservoirs of C. coli [7].

Pigs are often sub-clinically infected with Campylobacter spp. and contamination of meat during meat processing remains an important food safety risk $[3,4,8]$. Previous studies estimated the prevalence of contamination in pigs varying between $50 \%$ and $100 \%$, with excretion levels ranging from 102 to 107 Colony Forming Units (CFU) of Campylobacter per gram of faeces [9]. This study aimed at estimating the prevalence and levels of contamination of thermotolerant Campylobacter in faeces and carcasses of pigs during slaughtering. An evaluation of possible correlations between the genotypic and phenotypic expressions for resistance to antimicrobials in the isolated strains was performed. A comparison between the multi-locus sequence typing (MLST) profiles obtained from pig and human strains, isolated in the same time period, was also conducted.

\section{Materials and Methods}

\subsection{Study Design}

The sampling was carried out in a pig slaughterhouse located in the Abruzzo region of Italy. The sampling activities were carried out during sixteen sessions along the whole year, with four sessions for each season. A total of 12,308 animals were slaughtered, among which 280 animals were randomly selected ( 1 carcass for every 40-50 animals, about 17-18 animals sampled for each visit). This sample size was calculated to be able to estimate the prevalence of contamination with $6 \%$ of precision, considering $50 \%$ of expected prevalence and $95 \%$ of confidence level [10]. For all the animals sampled, the fattening phase of pigs was carried out in Italy, in 18 farms (coded F1 to F18) located in different regions of north (Piemonte, F3, F16, F9 and Emilia Romagna F1, F7, F10), central (Umbria F8, F12 and Abruzzo F2, F4, F5, F6, F11, F13, F15) and south Italy (Puglia F14, F17, F18) Figure 1. From each animal, the faecal content was taken immediately after the evisceration phase while swab samples from carcasses were collected before cooling, with a sampled surface of $400 \mathrm{~cm}^{2}$ for each carcass (withdrawal points: ham, back, belly, jowl). All samples were transported at $4{ }^{\circ} \mathrm{C}$ in refrigerated boxes and processed immediately on return to the laboratory.

\subsubsection{Campylobacter Culture and PCR Typing}

Faecal samples were cultured in Preston broth (Biolife, Milan, Italy) and incubated under microaerophilic conditions at $41.5^{\circ} \mathrm{C}$ for $24 \mathrm{~h}$. After incubation, 100 microliters of pre-enrichment broth were plated in duplicate on mCCDA and Karmali plates and incubated under microaerobic conditions at $41.5^{\circ} \mathrm{C}$ for $48 \mathrm{~h}$. Isolation and enumeration of thermotolerant Campylobacter were performed, respectively, according to part 1 and to the part 2 of the EN ISO 10272-2006 on swab samples. The isolates identified as Campylobacter spp. were then submitted to species identification by a multiplex PCR method, as previously described [11]. DNA was extracted using the Maxwell 16 Tissue DNA Purification Kit (Promega Corp., Madison, WI, USA) according to the manufacturer's instructions and quantified using a Nanodrop Spectrophotometer (Nanodrop Technologies, Celbio Srl., Milan, Italy).

\subsection{Pulsed-Field Gel Electrophoresis (PFGE)}

Pulsed-field gel electrophoresis (PFGE) was performed according to the instructions of the 2013 U.S. PulseNet protocol for Campylobacter [12]. C. coli strains were sub-cultured on Columbia agar 
at $41.5^{\circ} \mathrm{C}$ for $48 \mathrm{~h}$ in microaerophilic atmosphere and embedded in agarose blocks (Seakem Gold agarose, Lonza, Rockland, NY, USA). The blocks were then lysed, washed and digested with SmaI and KpnI enzymes (Promega, Italy), $25 \mathrm{U}$ at $25^{\circ} \mathrm{C}$ for $4 \mathrm{~h}$ and subjected to pulsed-field electrophoresis in $1 \%$ agarose gel (Seakem Gold agarose, Lonza) for $18 \mathrm{~h}$ (Chef Mapper XA, Biorad Laboratories, Hercules, CA, USA). Salmonella serovar Branderup H9812 digested with XbaI enzyme (Promega, Milan, Italy), was used as standard molecular weight size. The gel was stained with Sybr Safe DNA gel stain (Invitrogen) and photographed at transilluminator (Alpha Innotech). The image analysis was performed using the program Bionumerics v. 7.6 (Applied Maths NV, Sint-Martens-Latem, Belgium). Level of similarity was calculated with the Dice correlation coefficient (position tolerance was set at $1 \%$ ), and the unweighted pair group mathematical average UPGMA clustering algorithm was used for cluster analysis of the PFGE pattern. PFGE-clusters were defined at $100 \%$ similarity between macrorestriction patterns [13]. Untypeable isolates were not included in the analysis.

\subsection{Multi-Locus Sequence Typing (MLST)}

MLST was performed as described by Dingle et al. [14] for all C. coli isolates. MLST amplifies a segment of 7 housekeeping genes: aspA (aspartase, $477 \mathrm{bp}$ ), glnA (glutamine synthase, $477 \mathrm{bp}$ ), gltA (citrate synthase, 402 bp), glyA (serine hydroxyl methyl transferase, 507 bp), pgm (phosphor glucomutase, $498 \mathrm{bp}$ ), and tkt (transketolase, $459 \mathrm{bp}$ ) and uncA (ATP synthase, alpha subunit, $489 \mathrm{bp}$ ), to yield a total composite sequence length (all 7 loci) of $3309 \mathrm{bp}$. Oligonucleotides primers for the PCR and cycle sequencing reactions were carried out according to the Campylobacter MLST website [15]. Briefly, purified PCR products were sequenced by using the ABI PRISM BigDyeßTerminator 3.1 Cycle Sequencing Kit (Applied Biosystems) according to the manufacturer and analyzed with the ABI PRISM 3500 Genetic Analyzer (Applied Biosystems). The alleles, sequence types (STs) and clonal complexes (CCs) were identified using the MLST database available online [16]. Novel alleles were submitted to the PubMLST C. jejuni/C. coli databases curators for number assignment. A minimum spanning tree (MST) of the results was generated in PHILOVIZ 2.0 [17] using the goeBURST algorithm [18]. In this phylogenetic tree construction, the sequences of the seven house-keeping genes analyzed (including MLST allelic sequences and flanking regions) were aligned by MUSCLE in MEGA 5.0 [19]. A phylogenetic tree was built using the maximum parsimony method and was analysed by 1000 replicates in the bootstrap test.

\subsection{Antimicrobial Susceptibility and Resistance Genes}

Susceptibility to antimicrobials was evaluated with the microdilution method using the Sensititre automated system (TREK Diagnostic Systems, Italy) following the harmonised rules for the monitoring and reporting of AMR in Europe (Commission Implementing Decision 2013/652/EC). Colonies were cultured on Columbia agar for $48 \mathrm{~h}$ in microaerophilic atmosphere, inoculated in Mueller Hinton Broth supplemented with blood and dispensed into Eucamp microtiter plates (TREK Diagnostic Systems, Biomedical Service, Italy), containing known scalar concentrations of the following antimicrobial substances: gentamicin (GEN) $(0.12-16 \mu \mathrm{g} / \mathrm{mL})$, streptomycin (STR) $(1-16 \mu \mathrm{g} / \mathrm{mL})$, ciprofloxacin (CIP) $(0.06-4 \mu \mathrm{g} / \mathrm{mL})$, tetracycline (TET) $(0.25-16 \mu \mathrm{g} / \mathrm{mL})$, erythromycin (ERY) $(0.5-32 \mu \mathrm{g} / \mathrm{mL})$, nalidixic acid (NAL) $(2-64 \mu \mathrm{g} / \mathrm{mL})$, and chloramphenicol (CHL) $(2-32 \mu \mathrm{g} / \mathrm{mL})$. The plates were then incubated at $42{ }^{\circ} \mathrm{C}$ in microaerobic atmosphere for $24 \mathrm{~h}$. To evaluate the MICs of the isolates, Swin v3.3 Software (Thermo Fisher Scientific) was used in accordance with the epidemiological cutoff values (ECOFFs) as defined by EUCAST (European Committee on antimicrobial breakpoints) (www.eucast.org) to interpret their antimicrobial susceptibilities. C. jejuni strain NCTC 11351 was included for the quality control of the minimal inhibitory concentration (MIC) test. Strains were considered resistant when MIC break points were $\geq$ to $0.5 \geq$ to $8, \geq$ to $2, \geq$ to $16, \geq$ to 4 and $\geq$ to 2 , for respectively, ciprofloxaxin, erytromycin, gentamicin, nalidixid acid, streptomycin and tetracycline. C. coli genome assemblies, available at the NRL for Campylobacter, were searched for genomic AMR traits presence. AMR genes were identified in silico using ABRicate v. 0.8 (Available online: https://github.com/ tseemann/abricate/) and by querying 
the publicly available Comprehensive Antibiotic Resistance Database [20]. Assemblies were annotated using Prokka v1.13 [21] and gyrA sequences were extracted using the query_pan_genome function in Roary v3.12.0 [22]. GyrA genes were aligned using Uniprot UGENE v1.18.0 [23]. Only mutations in the quinolone resistance-determining region (QRDR) of gyr $A$ were considered to be the determinants of resistance, being these loci linked with phenotypic resistance to quinolones. In detail, for $g y r A$, we analyzed the amino acid changes at position 86 .

\section{Results}

A total of 280 pig carcasses were sampled at the slaughterhouse coming from different part of Italian Regions (Figure 1); the number of pigs finally sampled per farm was 10, 48, 7, 10, 10, 33, 12, 2, 1, 6, 2, 3, 6, 5, 1, 4, 1, 1 for farm F1, F2, F3, F4, F5, F6, F7, F,8, F9, F10, F11, F12, F13, F14, F15, F16, F17 and F18.

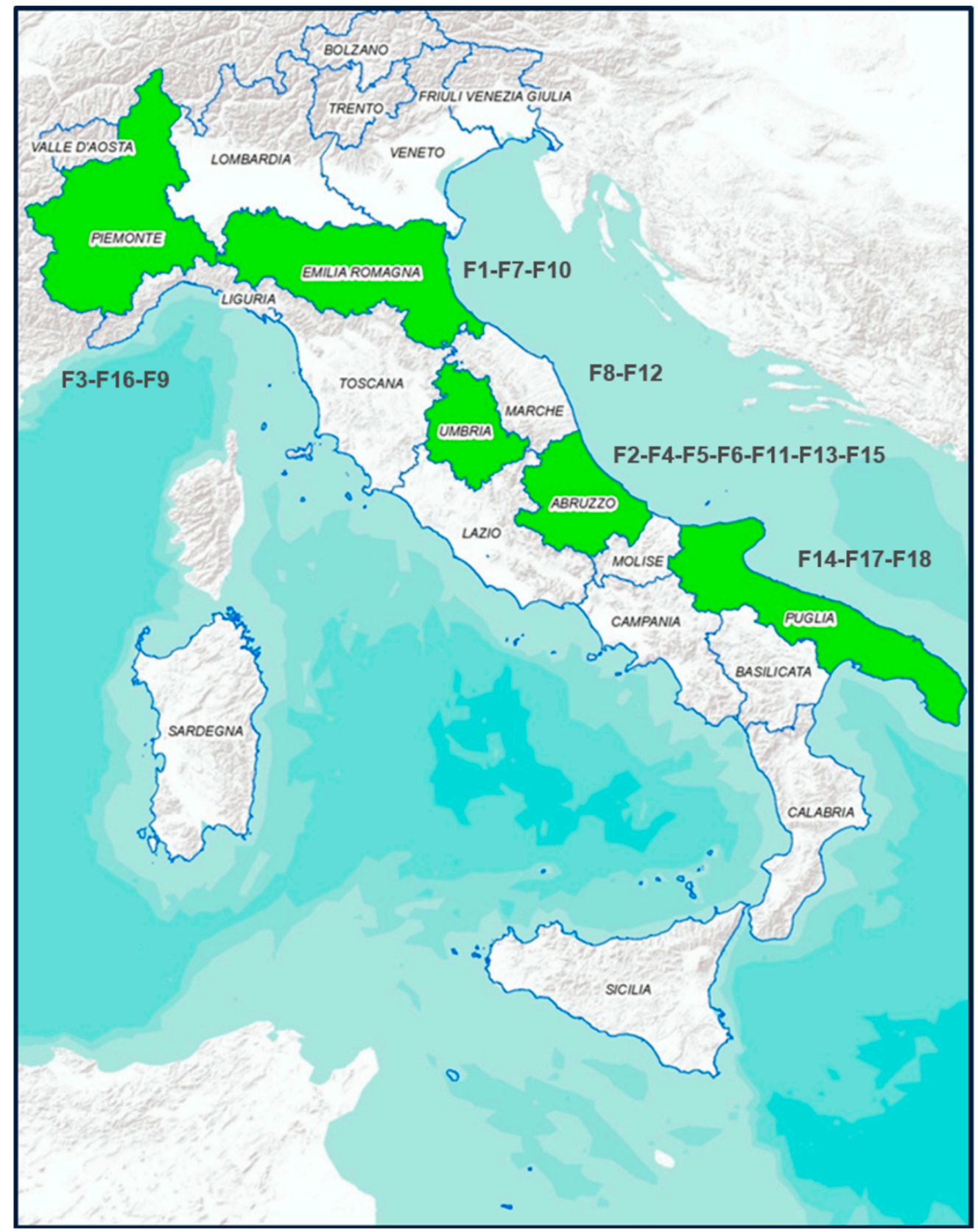

Figure 1. In green are the Italian Regions where pigs were bred. 
Among the 280 pigs, 162 females $(10.7 \%)$ and 118 males $(89.3 \%)$ and were represented by 216 fat $(77.14 \%)$ and 64 lean (22.86\%). Almost all the animals (93.57\%) were born in Italy, while $6.43 \%$ originated from other European countries but were fattened in Italy. Campylobacter has been isolated from both carcass swabs and faeces taken directly from intestinal loops of slaughtered animals. The prevalence of contamination was higher on carcasses (50.4\%, 95\% C.I.: $44.5 \%-56.2 \%)$ than in faeces (32.9\%, 95\% C.I.: $27.6 \%-38.6 \%$ ). This difference was statistically significant ( $\chi^{2} \mathrm{McNemar} 14.2$, $p=0.002)$ during all the seasons. Only in summer, the prevalence of $C$. coli contamination was higher in faeces than in carcasses, as shown in Figure 2. Campylobacter contamination was low on carcasses: less than $10 \mathrm{cfu} / \mathrm{cm}^{2}$ in $90.8 \%$ of samples, between $10-50 \mathrm{cfu} / \mathrm{cm}^{2}$ and between $100-150 \mathrm{cfu} / \mathrm{cm}^{2}$ in $7.8 \%$ and $1.4 \%$ of samples, respectively. All positive animals were contaminated with C. coli, and three animals were also positive for C. jejuni. A total of $221 \mathrm{C}$. coli was isolated from swabs and faeces samples collected on pigs at slaughtering. A high genetic diversity for $C$. coli was observed, with both typing methods. PFGE profiles of C. coli strains, after SmaI and KpnI enzyme digestion, resulted in 25 clonal populations, according to a similarity of $90 \%$. The most representative are shown in Table 1 . In addition, during the same period, $C$. coli was isolated from 11 gastroenteritis hospitalized patients in the Abruzzo region. MLST analysis of 173 strains (162 from pigs and 11 from humans) of $C$. coli is shown in Figure 3. Almost all human isolates (90.90\%) and 96/162 of swine isolates (64.19\%) were assigned to the ST-828 complex. Among the pig isolates, the most frequent STs were ST-854 (16.04\%), ST-9264 (10.49\%) ST-1016 (6.8\%) and ST-1108 (5.55\%), as shown in Figure 3. Sequence types ST-828 $(4.05 \%)$, ST-829 $(2.31 \%)$, ST-1055 and ST-827 $(1.73 \%)$ were present both in human and pig isolates, as shown in Figure 3. Each analyzed farm was characterized by different STs and nine of them by seven different PFGE pulsotypes (P1-P7), as shown in Table 1. In particular, farm 2, showed the presence of 10 different STs followed by farm 6 with 7 STs, as shown in Table 1. It is apparent that some ST profiles of $C$. coli appear to dominate in a geographic area for a variable period. For example, some genotypes, such as ST-1617, ST-9264, ST-1016, ST-828 and ST-1108, were isolated in subsequent seasons in animals coming from the same farms, as shown in Table 1. On the other hand, the same ST was also found on different farms and, with the exception of two of these cases (ST-9264 and 854-ST), the isolates had the same PFGE pulsotype. This is the case of ST-1016 predominant in five farms (F2-F3-F4-F5-F6) with PFGE pulsotype P2; ST-1617 and ST-1108, respectively, common in three farms (F1, F2, F6) and (F2, F6 and F10) with PFGE pulsotypes P1 and P7. Table 1. ST-9264 and ST-854 were found to be the most diverse ST with two different PFGE types. In many animals, the same ST was isolated in both the faeces and the carcass, as shown in Table 1. The comparison of the STs by country of origin of the animals showed the presence of the same STs in the majority of cases, although distinct STs between animals born in Italy and abroad were found in $27.6 \%$ of the pigs.

The highest level of antimicrobial resistance of $C$. coli isolated from pigs was observed against quinolones and fluoroquinolones (74.66\% of isolates for nalidixic acid and 70.13\% for ciprofloxacin) together with aminoglycosides (90.95\% of isolates for streptomycin) and tetracyclines $(90.95 \%$ of isolates), as shown in Figure 4. Only $37.55 \%$ and $25.79 \%$ of the strains from pigs were resistant to Erytromycin and Gentamycin, respectively. C. coli strains isolated from humans showed similar antimicrobial resistance patterns: all isolates were resistant to quinolones and fluoroquinolones and $90.90 \%$ to tetracycline. Resistance to erythromycin was also quite high in both pigs (37.55\%) and clinical isolates $(20.0 \%)$. Streptomycin is revealed statistically more resistant in pig with respect to humans. Many strains were multi-resistant with a predominant R-type TeSCipNa (56.5\%). The analysis of the isolates allowed to identify several resistance genes that include: gyrA, tet $(\mathrm{O}), \mathrm{cmeA}, \mathrm{cmeB}, \mathrm{cmeC}$ and cmeR. The study of genotypic resistance by sequencing the QRDR region of the gyrA gene showed the presence of specific mutations responsible for quinolone and fluoroquinolone resistance in the genome of all human isolates and in $64.81 \%$ of the pig isolates. A different percentage was observed for bacterial strains containing tet $(\mathrm{O})$ gene, identified in $72.72 \%$ and in $88.88 \%$ of human and pig isolates, respectively. The CmeABC multidrug efflux pump with its $\mathrm{CmeR}$ regulator gene was identified in every human and pig strain analysed, covering a range between $99.38 \%$ and $100 \%$. Strong correlations 
between phenotypic and genotypic resistance were found for fluoroquinolones and tetracycline in pig and human isolates. In particular, we observed a high level of concordance for the two resistance rates for gyrA $(92.41 \%$ and $100 \%)$ and tet $(\mathrm{O})(97.70 \%$ and 80.8$)$, in swine and clinical cases, respectively.

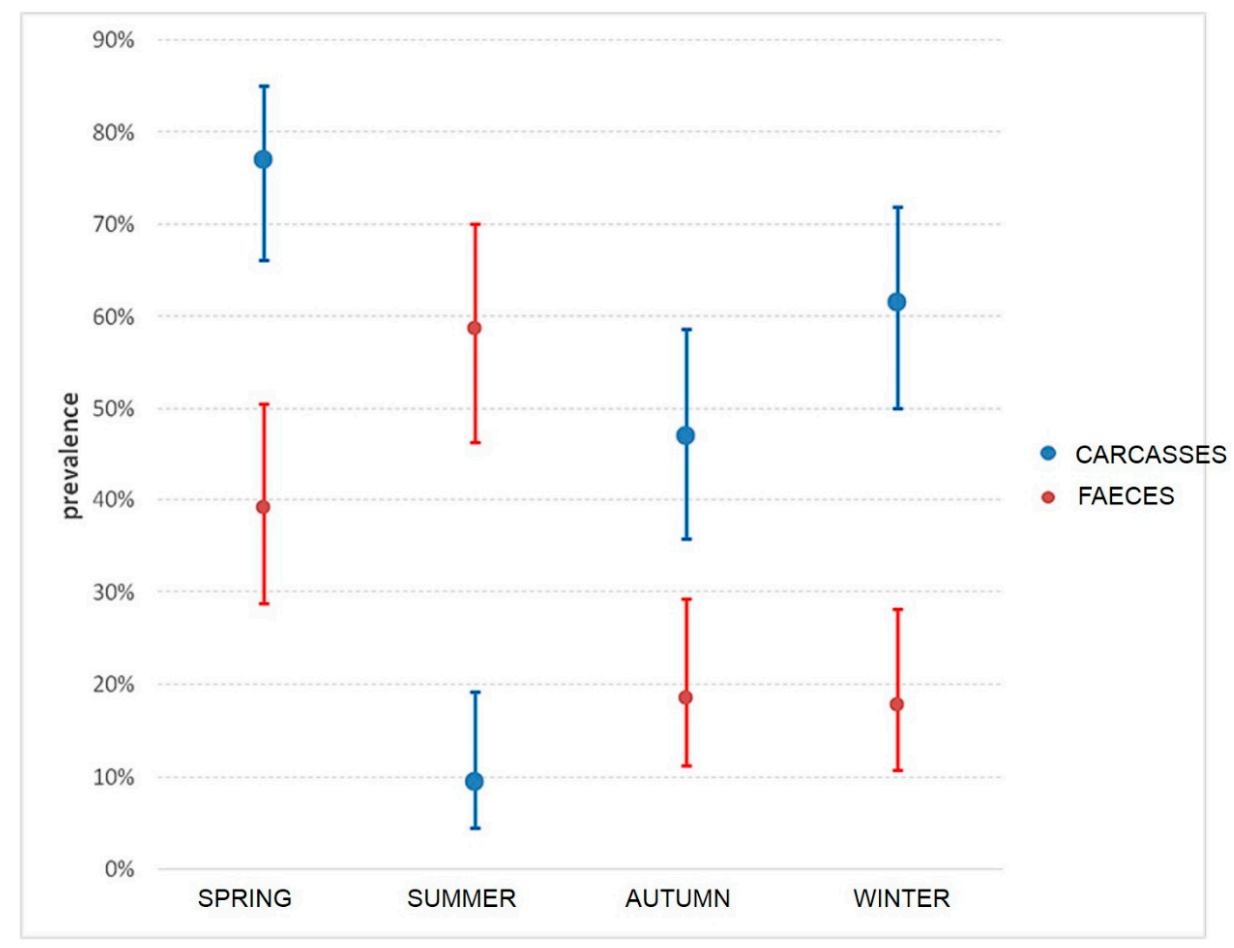

Figure 2. Prevalence and CI in carcasses and faeces during seasons.

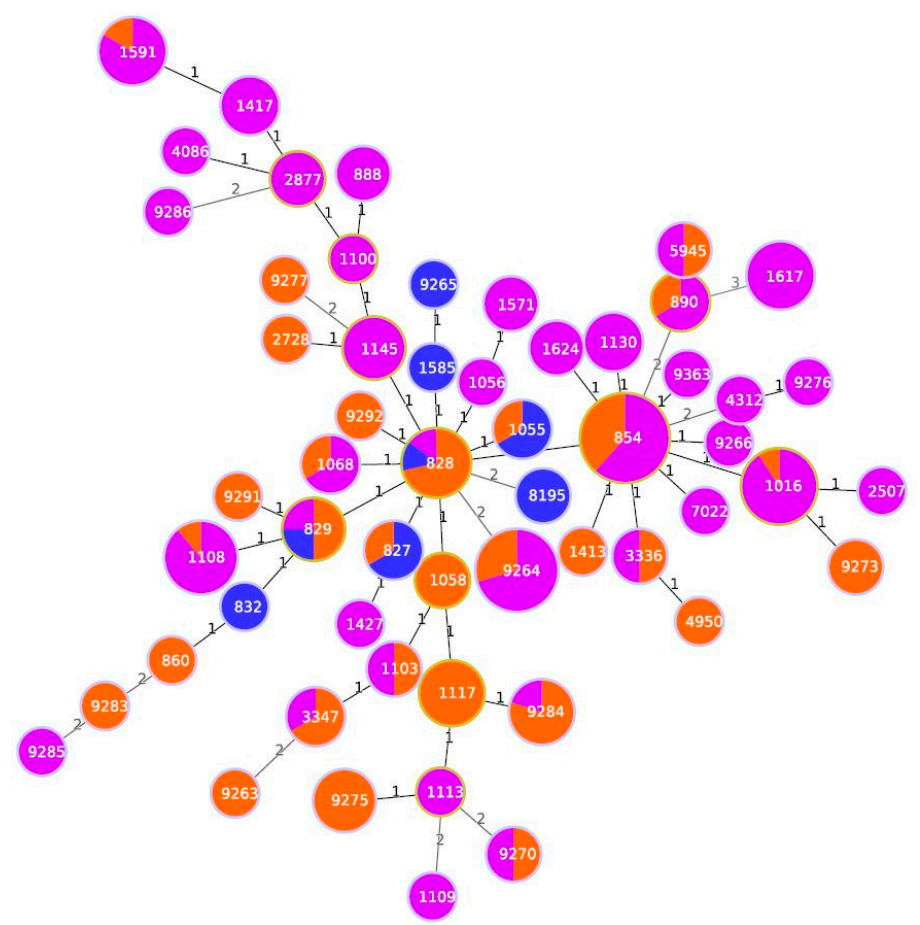

Figure 3. Minimum spanning tree generated for 11 human isolates and 162 pig isolates. Each circle represents an allelic profile. The numbers on the connecting lines illustrate the numbers of target genes with differing alleles. The different sources are distinguished by the colors of the circles with blue for human isolates, orange for pig stool and violet for pig carcasses isolates. 
Table 1. The most representative Pulsed-field gel electrophoresis (PFGE) pulsotypes and multi-locus sequence typing (MLST) profiles from Campylobacter coli isolated from swine per farm.

\begin{tabular}{|c|c|c|c|c|c|c|c|c|c|c|}
\hline PFGE & $\begin{array}{l}\text { MLST } \\
\text { Profile }\end{array}$ & FARM 1 & FARM 2 & FARM 3 & FARM 4 & FARM 5 & FARM 6 & FARM 7 & FARM 9 & FARM 10 \\
\hline \multirow{3}{*}{ P1 } & ST-1617 & Winter (C) & Autumn, Spring (C) & l & l & l & Spring $(\mathrm{C})$ & & l & l \\
\hline & ST-9264 & Winter (C) & $\begin{array}{l}\text { Winter }(\mathrm{F})(\mathrm{C}) \text {, } \\
\text { Spring }(\mathrm{C})\end{array}$ & Spring $(\mathrm{C})$ & / & l & l & Spring $(\mathrm{F})(\mathrm{C})$ & / & l \\
\hline & ST- $1624 *$ & l & Spring $(\mathrm{C})$ & l & 1 & l & 1 & 1 & l & 1 \\
\hline $\mathbf{P 2}$ & ST-1016* & l & Autumn (C) & Spring $(\mathrm{C})$ & $\begin{array}{c}\text { Autumn }(C), \\
\text { Spring (C) }\end{array}$ & Autumn $(\mathrm{C})(\mathrm{F})$ & $\begin{array}{l}\text { Winter (C), } \\
\text { Spring (C) }\end{array}$ & / & l & l \\
\hline \multirow{4}{*}{ P3 } & ST-1591 & 1 & Spring $(\mathrm{C})(\mathrm{F})$ & l & I & l & l & / & l & 1 \\
\hline & ST-1417 & 1 & Spring $(\mathrm{C})$ & 1 & 1 & 1 & 1 & 1 & 1 & l \\
\hline & ST-9277 & 1 & l & l & 1 & 1 & Spring $(\mathrm{F})$ & l & 1 & 1 \\
\hline & ST-1113* & 1 & 1 & Spring $(\mathrm{C})$ & 1 & 1 & 1 & l & l & 1 \\
\hline \multirow{4}{*}{ P4 } & ST- $854 *$ & Winter (C) & Spring $(\mathrm{C})(\mathrm{F})$ & l & l & l & Spring $(C)(F)^{* *}$ & l & l & l \\
\hline & ST-9276 & 1 & l & l & l & l & Spring $(\mathrm{C})$ & 1 & l & / \\
\hline & ST-9275 & 1 & Summer (F) & l & 1 & l & l & 1 & 1 & l \\
\hline & ST-1130* & 1 & l & Spring $(\mathrm{C})$ & I & l & l & 1 & 1 & l \\
\hline \multirow{3}{*}{ P5 } & ST-1117* & l & Spring $(\mathrm{F})$ & l & l & l & l & l & l & l \\
\hline & ST-9264 & 1 & Winter $(F)$, Spring $(C)$ & l & l & l & I & Spring $(F)(C)$ & l & 1 \\
\hline & ST-9284 & 1 & l & l & l & l & 1 & Summer (F) & l & 1 \\
\hline \multirow{3}{*}{ P6 } & ST-9291 & 1 & l & l & l & 1 & l & 1 & Summer $(\mathrm{F})^{* *}$ & \\
\hline & ST-854 * & l & $\begin{array}{l}\text { Winter }(\mathrm{C}) \text {, } \\
\text { Spring }(\mathrm{F})^{* *}\end{array}$ & l & / & l & Spring $(C)(F)^{* *}$ & / & / & l \\
\hline & ST- $828 *$ & l & l & l & / & / & $\begin{array}{l}\text { Winter }(\mathrm{C}) \text {, } \\
\text { Spring }(\mathrm{F})^{* *}\end{array}$ & / & l & l \\
\hline \multirow{2}{*}{ P7 } & ST-1427* & Winter (C) & l & l & I & 1 & & I & I & 1 \\
\hline & ST-1108* & Winter (C) & / & l & l & l & $\begin{array}{l}\text { Summer }(\mathrm{F}), * * \\
\text { Winter }(\mathrm{C})^{* * *}\end{array}$ & / & l & Winter $(\mathrm{C})$ \\
\hline
\end{tabular}




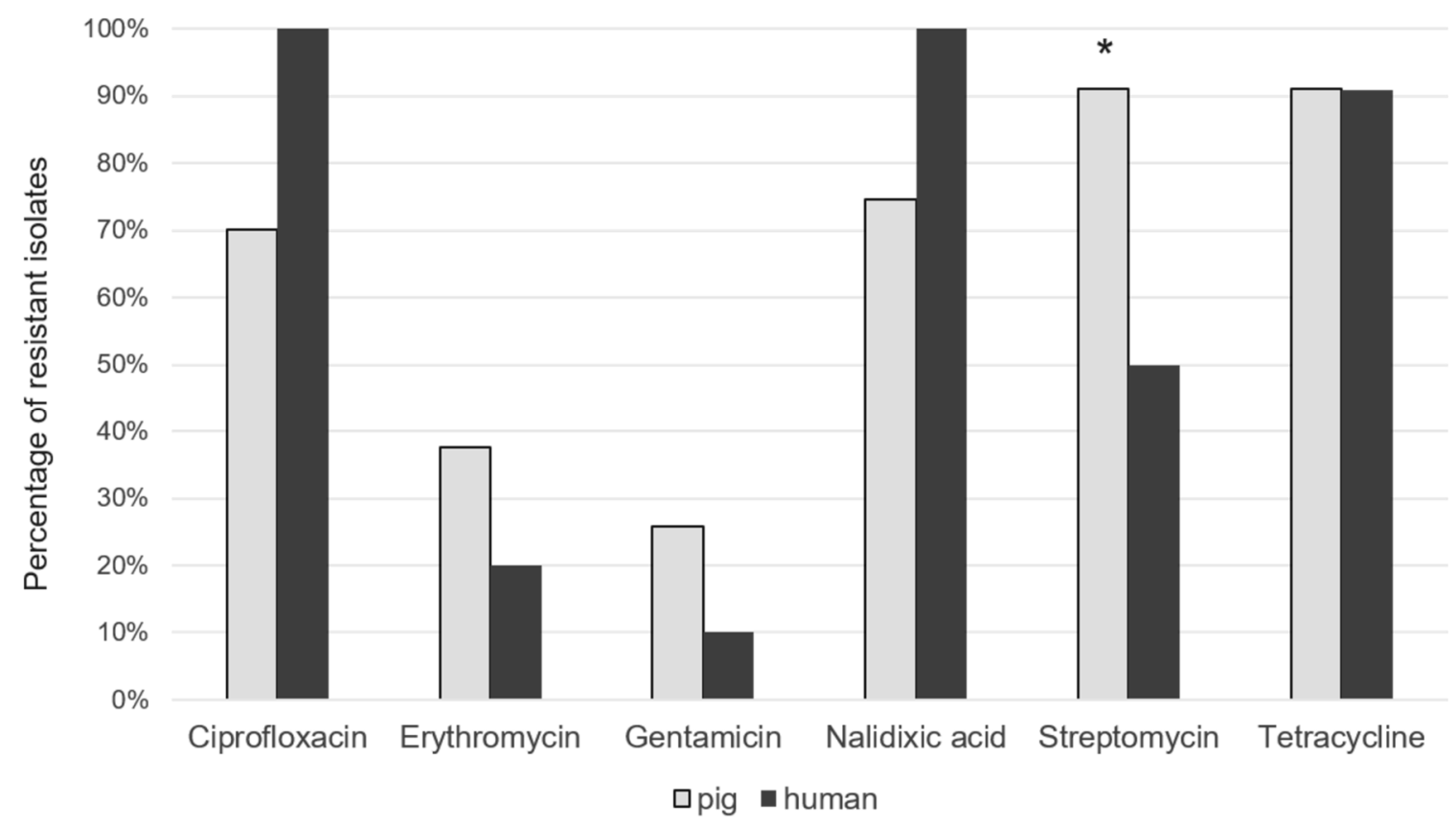

Figure 4. Percentage of resistant C. coli isolates from pig and humans. ${ }^{*} p \leq 0.5$.

\section{Discussion}

Pigs are considered a possible source of Campylobacter infection of humans, and the high percentage of contaminated pig carcasses in this study confirm the role of this animal species as one of the main animal reservoirs of $C$. coli $[24,25]$. Furthermore, C. coli shows high levels of antimicrobial resistance, thus representing an additional public health problem. A high prevalence of Campylobacter in pigs is frequently observed in many countries: levels from $0 \%$ to $92.7 \%$ were reported for European members and no member states [24,26-28]. Differences in sampling and analytical methods may explain these results. In this study, all isolates from pigs were found to be C. coli. A co-infection with C. jejuni has only been observed in three animals.

This is in agreement with a previous report from Italy, showing low levels of C. jejuni colonization in pigs [29]. The higher prevalence of Campylobacter in carcasses compared to faeces could indicate a cross contamination during the slaughter process, argued by the low level of contamination and also confirmed by PFGE analysis. The higher percentage of positive faeces recorded during the warm seasons are consistent with the Campylobacter seasonality reported in pig [30]. C. coli is often present in the intestinal tracts of swine, and carcasses may be contaminated during evisceration, which is considered the most critical phase of slaughter, with possible cross-contamination between carcasses, if they have the possibility to be in contact along the slaughtering line or the equipment used for evisceration is not properly cleaned and disinfected between carcasses [29]. However, contamination levels are significantly reduced by the chilling of the carcasses [31] and Campylobacter is found only at low levels in pork retail products [32-34]. Despite not be able to grow out of the body of the living host, Campylobacter can survive in food products at refrigeration temperatures from one to three weeks. This clearly indicates the importance of the contamination of carcasses (and therefore of the meats), given the capacity of Campylobacter to survive after refrigeration and the following processing phases, and determining infections in the consumers [29]. Each farm from which the tested pigs originated resulted as characterized by different clonal populations, characterized by several STs. It is noteworthy that some of these STs, circulating in different farms and seasons, show a specific spatial and temporal distribution. In particular, ST-9264 was present in three different farms. In detail, in farm 2, this clone was isolated in winter in faeces and carcasses, demonstrating a contamination of the carcasses from the faeces; furthermore, it has also been isolated in the carcasses during the following season, therefore showing a temporal resistance. 
The ST-1016 828 complex was isolated in the same farms on carcasses of animals in two following seasons, while it was isolated in three other farms during one season, suggesting that this could be a strain adapted to pigs. ST-9291 was isolated in summer in one farm from faeces of animals reared in central Italy but born in HU, suggesting that this could be a strain introduced from abroad. There was no similarity between strains isolated from different lots, indicating that there was no persistence of strains in the slaughterhouse. These populations were prevalent in different seasons and have been isolated from animals born, raised and fattened on holdings in different Italian regions. The seasonality of the blocks and the link to the different farms seems to be confirmed for some strains. Several studies described the antimicrobial resistance of Campylobacter isolates from pigs [35]. The results of this study apparently showed higher percentages of antimicrobial resistance against ciprofloxacin, nalidixic acid, tetracycline and streptomycin, than those observed in other EU countries. In this study, C. coli strains resulted highly resistant to tetracyclines (90.95\%), streptomycin (90.95\%), and to quinolone (ciprofloxacin and nalidixic acid, $70.13 \%$ and 74.66 of the isolates, respectively). Macrolides, important compounds for the treatment of human campylobacteriosis, showed relevant levels of resistance $(37.55 \%)$. In C. jejuni, tet.(O) was plasmid-encoded in $54 \%$ of tetracycline-resistant isolates, whereas in $C$. coli, tet $(\mathrm{O})$ appeared to be located on the chromosome [35-37]. The major mechanism of tetracycline resistance in Campylobacter spp. is the binding and protection of ribosomal A site by the protein Tet(O). Ciprofloxacin resistance in C. jejuni and C. coli is mainly due to point mutations in the quinolone resistance-determining region (QRDR) of the GyrA protein [38]. Erythromycin resistance in Campylobacter spp. has been associated with mutations in the 23S rRNA and in the large loop of the L4 and L22 50S ribosomal proteins [39]. Multidrug efflux can also contribute to reducing the intracellular concentration of several antibiotics, including tetracycline, ciprofloxacin and erythromycin [38]. In the EU, the proportion of C. coli isolates resistant to both ciprofloxacin and erythromycin (used in human campylobacteriosis) is low (10.2\%), although Portugal, Spain and Finland reported in 2017 high proportions of strains with combined resistance to these two antimicrobials. An increasing proportion of $C$. coli isolates resistant to ciprofloxacin and tetracyclines was observed in many countries from 2014 to 2017. Thirteen EU countries reported in 2017 a proportion of ciprofloxacin-resistant C. coli isolated varying between $70.5 \%$ and $100 \%$. High levels of resistance to ciprofloxacin (52.3\%) and tetracyclines $(51.5 \%)$ were also observed in C. coli from fattening pigs, with a lower percentage for erythromycin (15.6\%). Combined resistance to ciprofloxacin and erythromycin was reported in $61.2 \%$ of C. coli isolates from fattening pigs in Spain [40]. In Italy, high rates of ciprofloxacin and tetracycline resistance in Campylobacter spp. have been observed and an increasing percentage of $C$. coli strains simultaneously resistant to ciprofloxacin, tetracycline and erythromycin have been found [41]. In our study, we observed that $C$. coli strains of human origin showed a higher resistance than those recovered from pigs and primarily for ciprofloxacin and nalidixic acid (100\%); many strains were resistant to erytromicyn $(20 \%)$. Multi-resistance, defined as resistance to antimicrobials belonging to at least three different classes of antibiotics, was found among 65\%, and the predominant R-Type was TETSTRCIPNAL. We have characterized C. coli isolated for the presence of resistance genes, and the results of phenotypic and genetic analyses of resistance to tetracycline were fully concordant. All of the strains resistant to tetracycline were shown to carry the gene tet $(\mathrm{O})$ responsible for the synthesis of protein Tet $(\mathrm{O})$, which abolishes the inhibitory effect of tetracycline on protein synthesis by a non-covalent modification of the ribosomes [41]. In our study, we noted the presence of the tet (O) gene in $88.88 \%$ of isolated pigs, indicating a very strong correlation $(98.76 \%)$ between phenotypic and genotypic resistances, which decrease to the $80 \%$ level of concordance in humans. The high resistance to tetracycline of bacteria isolated from food, including Campylobacter, remains a serious problem in many European countries [40]. Fluoroquinolone resistance in our strains was mainly due to the presence of the Thr86Ile GyrA mutation. This mutation is the most prevalent in clinical and also in veterinary isolates [35,38]. In our study, it was present in $64.81 \%$ and $100 \%$ of the isolates from pigs and humans, respectively, displaying a high degree of correlation among the two types of resistances of $96.80 \%$ and $100 \%$. Multiple mechanisms associated with antibiotic resistance have been 
identified in Campylobacter spp., but target mutation and drug efflux are most relevant to the resistance to fluoroquinolones and macrolides, as reported in previous studies [42,43]. These two mechanisms function together in conferring a high level of resistance to the two classes of antibiotics. Expression of cmeABC is subject to regulation by $\mathrm{CmeR}$, a repressor encoded by a gene immediately upstream on cmeA [43]. CmeABC, a multidrug efflux system in C. jejuni, plays an important role in the resistance to different antimicrobials and toxic compounds and it is present also in C. coli [44], as reported in other studies [45]. In our study, it was present in all pig and human isolates we analyzed.

\section{Conclusions}

In conclusion, pigs may play a role as an underestimated reservoir of potentially pathogenic Campylobacter strains for humans. These animals could contribute to human campylobacteriosis cases and outbreaks through consumption of contaminated meat. The results obtained also provide evidence that antimicrobial resistance is common among Campylobacter strains isolated from pigs in Italy, thus indicating the need for continued monitoring and application of reduction strategies within these meat-producing animals. The WHO priority list suggests that the prioritization of research and development of new antibiotics against multidrug-resistant tuberculosis and Gram-negative bacteria is urgently needed. Global research and development strategies should also include antibiotics active against more common community bacteria, such as antibiotic-resistant Salmonella spp, Campylobacter spp, and Helicobacter pylori [42].

Author Contributions: Conceptualization, E.D.G., G.D.D., and P.C.; data curation, G.D.D., D.D.S. and F.M.; formal analysis, F.M. and D.N.; funding acquisition, E.D.G.; investigation, G.D.D.; project administration, E.D.G.; resources, F.M., K.Z. and D.N.; supervision, R.N., E.D.G., P.C.; writing一original draft, G.D.D., F.M; writing一review and editing, E.D.G., P.C., and F.M. All authors have read and agreed to the published version of the manuscript.

Funding: The work was supported by funding from the Italian Ministry of Health

Conflicts of Interest: The authors declare no conflicts of interest.

\section{References}

1. EFSA and ECDC (European Food Safety Authority and European Centre for Disease Prevention and Control). The European Union summary report on trends and sources of zoonoses, zoonotic agents and food-borne outbreaks in 2017. EFSA J. 2018, 16, e05500. [CrossRef]

2. Jacobs-Reitsma, W. Campylobacter in the food supply. In Campylobacter, 2nd ed.; Nachamkin, I., Blaser, M.J., Eds.; ASM Press: Washington, DC, USA, 2000; pp. 467-481.

3. Gillespie, I.A.; O’Brien, S.J.; Frost, J.A.; Adak, G.K.; Horby, P.; Swan, A.V.; Painter, M.J.; Neal, K.R. A case-case comparison of Campylobacter coli and Campylobacter jejuni infection: A tool for generating hypotheses. Emerg. Infect. Dis. 2002, 8, 937-942. [CrossRef] [PubMed]

4. Tam, C.C.; O’Brien, S.J.; Adak, G.K.; Meakins, S.M.; Frost, J.A. Campylobacter coli-An important foodborne pathogen. J. Infect. 2003, 47, 28-32. [CrossRef]

5. Hermans, D.; Pasmans, F.; Messens, W.; Martel, A.; Van Immerseel, F.; Rasschaert, G.; Heyndrickx, M.; Van Deun, K.; Haesebrouck, F. Poultry as a host for the zoonotic pathogen Campylobacter jejuni. Vector Borne Zoonotic Dis. 2012, 12, 89-98. [CrossRef] [PubMed]

6. Leatherbarrow, A.J.; Cripps, P.J.; Diggle, P.J.; French, N.P. Temporal and farm-management-associated variation in the faecal-pat prevalence of Campylobacter jejuni in ruminants. Epidemiol. Infect. 2010, 138, 549-558.

7. Kempf, I.; Kerouanton, A.; Bougeard, S.; Nagard, B.; Rose, V.; Mourand, G.; Osterberg, J.; Denis, M.; Bengtsson, B.O. Campylobacter coli in Organic and Conventional Pig Production in France and Sweden: Prevalence and Antimicrobial Resistance. Front. Microbiol. 2017, 8, 955. [CrossRef]

8. Niederer, L.; Kuhnert, P.; Egger, R.; Büttner, S.; Hächler, H.; Korczak, B.M. Genotypes and antibiotic resistances of Campylobacter jejuni and Campylobacter coli isolates from domestic and travel-associated human cases. Appl. Environ. Microbiol. 2012, 78, 288-291. [CrossRef] 
9. Alter, T.; Gaull, F.; Kasimir, S.; Gurtler, M.; Mielke, H.; Linnebur, M.; Fehlhaber, K. Prevalences and transmission routes of Campylobacter spp. strains within multiple pig farms. Vet. Microbiol. 2005, 108, 251-261. [CrossRef]

10. Thrushfield, M. Veterinary Epidemiology, 3rd ed.; Blackwell Science Ltd.: Hoboken, NJ, USA, 2007; p. 610.

11. Di Giannatale, E.; Di Serafino, G.; Zilli, K.; Alessiani, A.; Sacchini, L.; Garofolo, G.; Aprea, G.; Marotta, F. Characterization of antimicrobial resistance patterns and detection of virulence genes in Campylobacter isolates in Italy. Sensors 2014, 14, 3308-3322. [CrossRef]

12. Centers for Disease Control and Prevention, CDC. Standard Operating Proce-dure for PulseNet PFGE of Campylobacter Jejuni, p. 12. PNL03 Last Updated April 2013. Available online: http://www.cdc.gov/ pulsenet/PDF/campylobacter-pfge-protocol-508c.pdf (accessed on 7 January 2015).

13. Grothues, D.; Tümmler, B. New approaches in genome analysis by pulsed-field gel electrophoresis: Application to the analysis of Pseudomonas species. Mol. Microbiol. 1991, 5, 2763-2776. [CrossRef]

14. Dingle, K.E.; Van Den Braak, N.; Colles, F.M.; Price, L.J.; Woodward, D.L.; Rodgers, F.G.; Endtz, H.P.; Van Belkum, A.; Maiden, M.C. Sequence typing confirms that Campylobacter jejuni strains associated with Guillain-Barré and Miller-Fisher syndromes are of diverse genetic lineage, serotype, and flagella type. J. Clin. Microbiol. 2001, 39, 3346-3349. [CrossRef] [PubMed]

15. Pubmlst. Available online: http://pubmlst.org/campylobacter/ (accessed on 10 October 2019).

16. Pubmlst. Available online: https://pubmlst.org/bigsdb?db=pubmlst_campylobacter_seqdef\&page=profiles (accessed on 10 October 2019).

17. Francisco, A.; Vaz, C.; Monteiro, P.; Melo-Cristino, J.; Ramirez, M.; Carriço, J. PHYLOViZ: Phylogenetic inference and data visualization for sequence based typing methods. BMC Bioinform. 2012, 13, 87. [CrossRef]

18. Francisco, A.; Bugalho, M.; Ramirez, M.; Carriço, J. Global optimal eBURST analysis of multilocus typing data using a graphic matroid approach. BMC Bioinform. 2009, 10, 152. [CrossRef] [PubMed]

19. Tamura, K.; Peterson, D.; Peterson, N.; Stecher, G.; Nei, M.; Kumar, S. Molecular evolutionary genetics analysis using maximum likelihood, evolutionary distance, and maximum parsimony methods. Mol. Biol. Evol. 2011, 28, 2731-2739. [CrossRef] [PubMed]

20. Jia, B.; Raphenya, A.R.; Alcock, B.; Waglechner, N.; Guo, P.; Tsang, K.K. CARD: Expansion and model-centric curation of the comprehensive antibiotic resistance database. Nucleic Acids Res. 2017, 45, D566-D573. [CrossRef] [PubMed]

21. Seemann, T. Prokka: Rapid prokaryotic genome annotation. Bioinformatics 2014, 30, 2068-2069. [CrossRef]

22. Page, A.J.; Cummins, C.A.; Hunt, M.; Wong, V.K.; Reuter, S.; Holden, M.T. Roary: Rapid large-scale prokaryote pan genome analysis. Bioinformatics 2015, 31, 3691-3693. [CrossRef]

23. Okonechnikov, K.; Golosova, O.; Fursov, M. The UGENE team. Unipro UGENE: A unified bioinformatics toolkit. Bioinformatics 2012, 28, 1166-1167. [CrossRef]

24. Avrain, L.; Vernozy-Rozand, C.; Kempf, I. Evidence for natural horizontal transfer of tetO gene between Campylobacter jejuni strains in chickens. J. Appl. Microbiol. 2004, 97, 134-140. [CrossRef]

25. Milnes, A.S.; Stewart, I.; Clifton-Hadley, F.A.; Davies, R.H.; Newell, D.G.; Sayers, A.R.; Cheasty, T.; Cassar, C.; Ridley, A.; Evans, S.J.; et al. Intestinal carriage of verocytotoxigenic Escherichia coli O157, Salmonella, thermophilic Campylobacter and Yersinia enterocolitica, in cattle, sheep and pigs at slaughter in Great Britain during 2003. Epidemiol. Infect. 2008, 136, 739-751. [CrossRef]

26. Tadesse, D.A.; Bahnson, P.B.; Funk, J.A.; Thakur, S.; Morrow, W.E.M.; Wittum, T.; DeGraves, F.; Rajala-Schultz, P.; Gebreyes, W.A. Prevalence and antimicrobial resistance profile of Campylobacter spp. isolated from conventional and antimicrobial-free swine production systems from different U.S. regions. Foodborne Pathog. Dis. 2011, 8, 367-374. [CrossRef] [PubMed]

27. EFSA and ECDC (European Food Safety Authority and European Centre for Disease Prevention and Control). The European Union summary report on trends and sources of zoonoses, zoonotic agents and food-borne outbreaks in 2014. EFSA J. 2015, 13, 4329. [CrossRef]

28. Pezzotti, G.; Serafin, A.; Luzzi, I.; Mioni, R.; Milan, M.; Perin, R. Occurrence and resistance to antibiotics of Campylobacter jejuni and Campylobacter coli in animals and meat in northeastern Italy. Int. J. Food Microbiol. 2003, 82, 281-287. [CrossRef]

29. Milan, C.; De Moraes, T.P.; de Mattos Ferrasso, M.; Ebersol, C.N.; Neto, A.C.P.S.; Da Silva, É.F.; Timm, C.D. Campylobacter coli in Swine Slaughtering Flowchart and Research of cdt Genes. Acta Sci. Veter. 2017, 45, 6. [CrossRef] 
30. Denis, M.; Henrique, E.; Chidaine, B.; Tircot, A.; Bougeard, S.; Fravalo, P. Campylobacter from sows in farrow-to-finish pig farms: Risk indicators and genetic diversity. Vet. Microbiol. 2011, 154, 163-170. [CrossRef]

31. Zhao, C.; Ge, B.; De Villena, J.; Sudler, R.; Yeh, E.; Zhao, S.; White, D.G.; Wagner, D.; Meng, J. Prevalence of Campylobacter spp.; Escherichia coli, and Salmonella serovars in retail chicken, turkey, pork, and beef from the Greater Washington, DC, area. Appl. Environ. Microbiol. 2001, 67, 5431-5436. [CrossRef]

32. Duffy, E.A.; Belk, K.E.; Sofos, J.N.; Bellinger, G.R.; Pape, A.; Smith, G.C. Extent of microbial contamination in United States pork retail products. J. Food Prot. 2001, 64, 172-178. [CrossRef]

33. Linblad, M.; Lindmark, H.; Thisted Lamberdz, S.; Lindqvist, R. Microbiological Baseline Study of Swine Carcasses at Swedish Slaughterhouses. J. Food Prot. 2007, 70, 1790-1797. [CrossRef]

34. Wieczorek, K.; Osek, J. Characteristics and antimicrobial resistance of Campylobacter isolated from pig and cattle carcasses in Poland Polish. J. Vet. Sci. 2013, 16, 501-508.

35. Taylor, D.E.; Courvalin, P. Mechanisms of antibiotic resistance in Campylobacter species. Antimicrob. Agents Chemother. 1988, 32, 1107-1112. [CrossRef]

36. Dasti, J.; Groß, U.; Pohl, S.; Lugert, R.; Weig, M.; Schmidt, R. Role of the plasmid-encoded tet(O) gene intetracycline-resistant clinical isolates of Campylobacter jejuni and Campylobacter coli. J. Med. Microbiol. 2007, 56, 833-837. [CrossRef] [PubMed]

37. Iovine, N.M. Resistance mechanisms in Campylobacter jejuni. Virulence 2013, 4, 230-240. [CrossRef] [PubMed]

38. Hao, H.; Yuan, Z.; Shen, Z.; Han, J.; Sahin, O.; Liu, P.; Zhang, Q. Mutational and transcriptomic changes involved in the development of macrolide resistance in Campylobacter jejuni. Antimicrob. Agents Chemother. 2013, 57, 1369-1378. [CrossRef] [PubMed]

39. EFSA/ECDC. The European Union summary report on antimicrobial resistance in zoonotic and indicator bacteria from humans, animals and food in 2017. EFSA J. 2019, 17, 5598.

40. García-Fernández, A.; Dionisi, A.M.; Arena, S.; Iglesias-Torrens, Y.; Carattoli, A.; Luzzi, I. Human Campylobacteriosis in Italy: Emergence of Multi-Drug Resistance to Ciprofloxacin, Tetracycline, and Erythromycin. Front. Microbiol. 2018, 9, 1906. [CrossRef]

41. Bolton, D.; Patriarchi, A.; Fox, Á.; Fanning, S. A study of the molecular basis of quinolone and macrolide resistance in a selection of Campylobacter isolates from intensive poultry flocks. Food Control 2013, 30, 222-226. [CrossRef]

42. Marotta, F.; Garofolo, G.; Di Marcantonio, L.; Di Serafino, G.; Neri, D.; Romantini, R.; Sacchini, L.; Alessiani, A.; Di Donato, G.; Nuvoloni, R.; et al. Correction: Antimicrobial resistance genotypes and phenotypes of Campylobacter jejuni isolated in Italy from humans, birds from wild and urban habitats, and poultry. PLoS ONE 2019, 14, e0225231. [CrossRef]

43. Li, W.; Atkinson, G.C.; Thakor, N.S.; Allas, U.; Lu, C.C.; Chan, K.Y.; Tenson, T.; Schulten, K.; Wilson, K.S.; Hauryliuk, V.; et al. Mechanism of tetracycline resistance by ribosomal protection protein Tet(O). Nat. Commun. 2013, 4, 1477. [CrossRef]

44. Guo, B.; Lin, J.; Reynolds, N.L.; Zhang, Q. Contribution of the Multidrug Efflux Transporter CmeABC to Antibiotic Resistance in Different Campylobacter Species. Foodborne Pathog. Dis. 2010, 7, 77-83. [CrossRef]

45. Tacconelli, E.; Pezzani, M.D. Public health burden of antimicrobial resistance in Europe. Lancet Infect. Dis. 2019, 19, 4-6. [CrossRef]

(C) 2020 by the authors. Licensee MDPI, Basel, Switzerland. This article is an open access article distributed under the terms and conditions of the Creative Commons Attribution (CC BY) license (http://creativecommons.org/licenses/by/4.0/). 